\title{
PERSPECTIVE OPEN Van der Waals oxide heteroepitaxy
}

\author{
Ying-Hao $\mathrm{Chu}^{1,2,3}$
}

The research field of oxide heteroepitaxy suffers from the characteristics of misfit strain and substrate clamping, hampering the optimization of performance and the gain of fundamental understanding of oxide systems. Recently, there are demonstrations on functional oxides epitaxially fabricated on layered muscovite substrate. In these heterostructures, due to the weak interaction between substrate and film, they show the lattice of films close to bulk with excellent strictive properties, suggesting that these critical problems can be potentially solved by van der Waals oxide heteroepitaxy. In addition, by exploiting the transparent and flexible features of muscovite, such a heteroepitaxy can deliver new material solutions to transparent soft technology. In this paper, the history, development, and current status of van der Waals oxide heteroepitaxy are addressed and discussed. In the end, new research directions in terms of fundamental study and practical application are proposed to highlight the importance of this research field.

npj Quantum Materials (2017)2:67; doi:10.1038/s41535-017-0069-9

\section{INTRODUCTION}

Strong interactions among degrees of freedom such as lattice, charge, orbital, and spin as shown in the center part of Fig. 1 result in a broad spectrum of intriguing functionalities in oxides, such as high temperature superconductivity, colossal magnetoresistance, and multiferroicity. Thus, oxide materials are ubiquitous in modern science and technology due to their fascinating physical properties and study on oxides represents an important research direction of materials science. Recently, strongly correlated electron systems in oxides ${ }^{1}$ form a new playground for nextgeneration electronic devices. In the process of the minimization and integration of electronic devices, materials in thin-film form are more favorable. Thus, a lot of researchers have been enthusiastically devoted to comprehensive investigation and advanced modulation of these intriguing phenomena in oxide thin films, nanostructures, and interfaces. ${ }^{2}$

In order to achieve the best performance and acquire the fundamental understanding of oxide thin films, the fabrication of oxide heteroepitaxy has been developed. Originally, the heteroepitaxy was focused on the growth of high-quality films on singlecrystalline substrates. In this type of heteroepitaxy, a selection of suitable substrate with similar crystal structure and lattice parameters is very important to obtain high quality heteroepitaxy since the atoms of substrate and film are linked by a formation of strong chemical bondings. Later on, the technique has been extended to create artificial superlattice ${ }^{3}$ and self-assembled lateral heteroepitaxy ${ }^{4,5}$ as shown in the bottom right panel of Fig. 1, to obtain more functionalities of oxide heteroepitaxy. However, there are two critical characteristics of epitaxial growth. The first one is the epitaxial strain due to a lattice mismatch between film and substrate. The misfit strain can lead to a change of the ground state, an interdiffusion of chemical elements, or formations of defects accumulated at interfaces due to the accommodation of the strain energy. Since it can not be avoided in a heteroepitaxial system, there are numerous studies that exploit the advantages of misfit strain to engineer the functionalities of thin films. ${ }^{6,7}$ The other one is the substrate clamping effect. Since the film is strongly tied to the substrate by the formation of bonding, the lattice interaction of substrate and film has to be taken into account when an epitaxial film is deformed. This effect is essential especially in strictive materials, such as piezoelectrics and magnetostrictive materials. When external stimuli such as electric or magnetic fields are applied, the lattice responding to the external fields has to overcome this effect, which in general, degrades the performance of strictive materials in thin-film form.

In order to solve these problems, the realization of free-standing samples provides a feasible solution. The creation of a freestanding oxide film can be tracked down to the 1990s, when the technique of laser lift-off was used to transfer epitaxial GaN film from sapphire to Si substrates. ${ }^{8}$ The same idea has been employed to create free-standing ferroelectric films and then transfer them to other substrates. ${ }^{9}$ The problem of substrate clamping effect can be partially solved depending on the selection of new bonding material between substrate and film. Another approach to remove substrate clamping is the creation of nanostructure. ${ }^{10-12}$ As an increase on the aspect ratio of nanostructure, the contact area of film and substrate is decreased. Thus, more volume fraction of film is free to move and the clamping effect is reduced. Recently, a solution-removable layer has been used as a sacrificial layer between film and substrate. ${ }^{13-15}$ After the heteroepitaxial growth, this layer is removed by being soaked in a solution, leaving a freestanding film in the solution. Then, the free-standing samples can be transferred to any suitable substrates. So far, no direct growth of a heteroepitaxy can surmount these two problems simultaneously, setting up a critical barrier of epitaxial growth. Therefore, an alternative approach capable of overcoming aforestated constraints can expand the domain of the oxide heteroepitaxy.

van der Waals (vdW) epitaxy was reported by Koma as shown in the bottom left panel of Fig. $1{ }^{16-19}$ Currently, it serves as a

\footnotetext{
${ }^{1}$ Department of Materials Science and Engineering, National Chiao Tung University, Hsinchu 30010, Taiwan; ${ }^{2}$ Department of Electrophysics, National Chiao Tung University, Hsinchu 30010, Taiwan and ${ }^{3}$ Material and Chemical Research Laboratories, Industrial Technology Research Institute, Hsinchu 31040, Taiwan Correspondence: Ying-Hao Chu (yhc@nctu.edu.tw)
} 


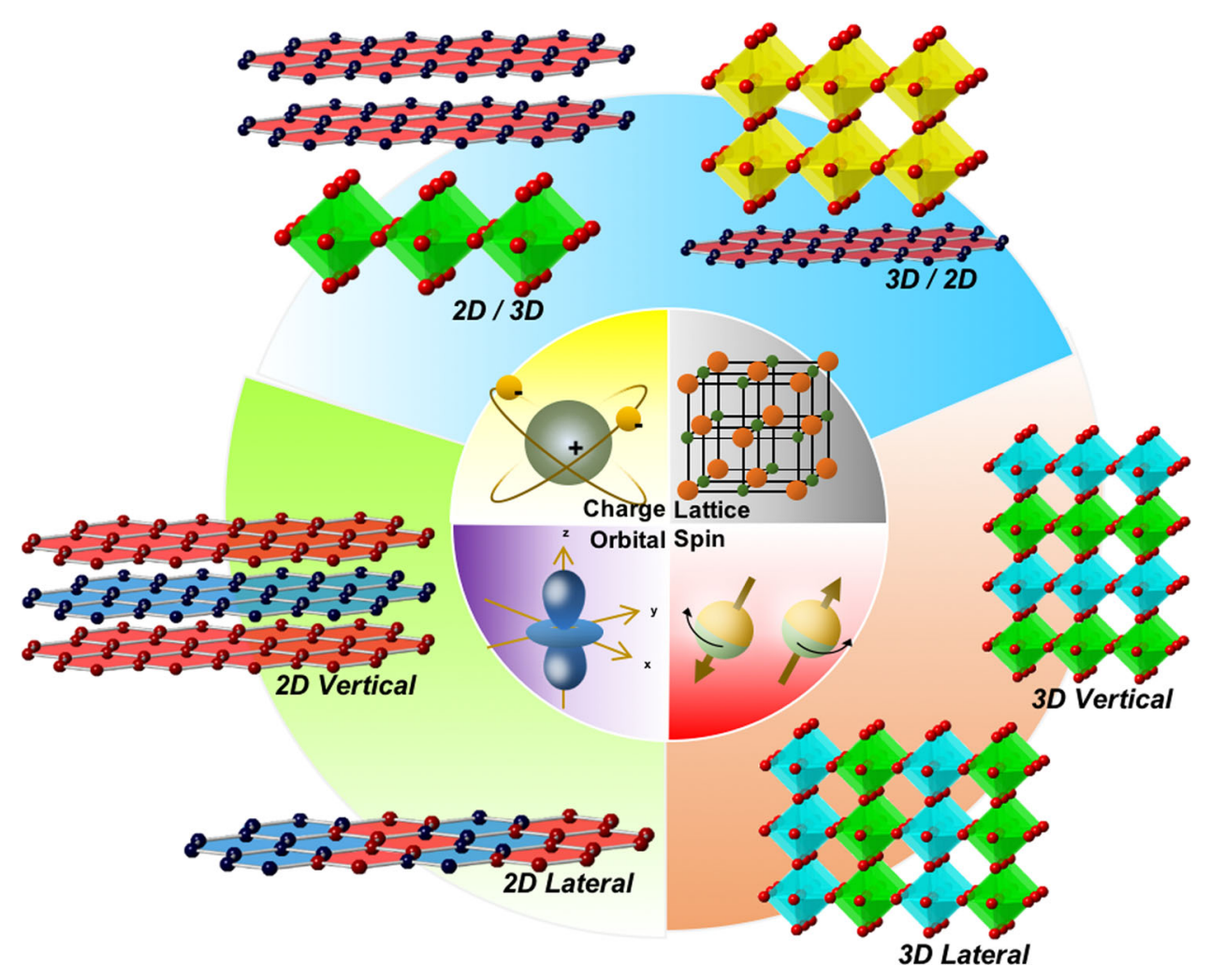

Fig. 1 Degrees of freedom and schematics of various heteroepitaxies

new paradigm of heteroepitaxy involving two-dimensional (2D)-layered materials with unique and promising properties. ${ }^{20-}$ ${ }^{23}$ The key feature of such an epitaxy involves weak vdW interactions between layers yielding high-quality films with a reduction of threading dislocations. In vdW epitaxy, the epilayer grows with its bulk lattice despite lattice mismatch as large as $60 \%{ }^{24}$ Therefore, epilayer has bulk single-crystal-like crystalline quality and the vdW interface is abrupt on an atomic scale without intermixing and chemical cross contamination. Recently, the lateral form of vdW has been also demonstrated with an abrupt interface, creating more possibilities of designing 2D heteroepitaxy. ${ }^{25}$ In principle, no stress or strain is induced by substrate across the contact plane in such a heteroepitaxy. Initially, vdW epitaxy strictly implied the growth of (2D) layered material on other (2D) layered substrates, but later it has been extended to the quasi-vdW epitaxy, the heteroepitaxial growth of (2D) layered materials onto $3 \mathrm{D}$ substrates or vice-versa as shown in the top panel of Fig. 1. The quasi-vdW epitaxy is intermediate to conventional and vdW epitaxies. The key advantage of this type of heteroepitaxy is the feature of weak interaction between film and substrate, resulting in a tolerance of lattice match and an almost strain-free sample. In order to apply such a concept for oxide heteroepitaxy, a search of layered oxides is of importance.

Typically oxides crystallize in structures based on the close packing of oxygen anions forming tetrahedral and octahedral sites occupied by cations. ${ }^{26}$ In oxides, one special family is silicates. According to the $\mathrm{Si}^{4+} / \mathrm{O}^{2-}$ radius ratio and the feature of $\mathrm{Si}-\mathrm{O}$ bond, a very strong preference for the formation of $\left(\mathrm{SiO}_{4}\right)^{4-}$ tetrahedra in silicates occurs. Depending on the atomic ratio of $\mathrm{Si}^{4+} / \mathrm{O}^{2-}$, a series of silicate structures are formed, including completely interconnected tetrahedra, sheets, chains, and rings. Silicate structures with sheets, such as mica, kaolinite, and montmorillonite, are layered materials. Micas are the most abundant subgroup of phyllosilicates. As shown in Fig. 2, the structural unit of layered mica $(\sim 1 \mathrm{~nm})$ contains two silicate tetrahedral sheets $\left(\mathrm{SiO}_{4}\right)$ on either side of a sheet based on aluminum octahedron $\left(\mathrm{AlO}_{6}\right)$ and these layer stacks are held together by interlayer cations. The tetrahedral sheet consists of honeycomb arrangement of hexagons of $\mathrm{SiO}_{4}$ tetrahedra such that each tetrahedron shares its basal oxygens with the adjacent tetrahedron. Si ions of these layers are partially replaced by Al ions $(1 / 3)$ to give a net negative charge. The apical oxygens together with hydroxyl group lying below the center of $\mathrm{SiO}_{4}$ hexagon of tetrahedral layer form the octahedral layer. These sandwichedlayer units with strong covalent bonding within them are weakly held together by interlayer cations resulting in a vdW gap. Cleavage of muscovite $\left(\mathrm{KAl}_{2}\left(\mathrm{Si}_{3} \mathrm{Al}\right) \mathrm{O}_{10}(\mathrm{OH})_{2}: \mathrm{a}=5.33 \AA, \mathrm{b}=9.23 \AA\right.$, $c=10.22 \AA$, and $\beta \sim 100^{\circ}$ ) along this vdW gap layer produces two large, atomically flat surfaces, with equal but randomly distributed $\mathrm{K}^{+}$atoms preserving charge neutrality, making muscovite mica an ideal substrate for vdW heteroepitaxy. ${ }^{27}$ The surface of mica provides an interesting playground for the study of 2D ion diffusion, 2D crystal nucleation, surface conductivity, molecular surface phenomena of adhesion, friction, and colloidal interactions. $^{28}$

Gold thin film on muscovite substrate was an early demonstration of a heteroepitaxy with mica substrate. ${ }^{29}$ Recently, due to a quick rise of the research on $2 \mathrm{D}$ layered materials, more materials were studied. For example, epitaxial $\mathrm{Bi}_{2} \mathrm{Te}_{3}$ thin films have been prepared on muscovite as a potential solution for transparent conducting electrode. ${ }^{30}$ Following up, oxide materials were extensively explored. ${ }^{31-33}$ Currently, most common oxide structures including rocksalt, wurtzite, perovskite, spinel, and antifluorite, were demonstrated in a heterostructure with determined epitaxial relationships as shown in the summary table of Fig. 2. Since there is no strong chemical bonding between substrate and film, the quality of van der Waals oxide heteroepitaxy depends on the competition of surface energy and the coherency of the symmetry between muscovite and film. Typically, three- or six-fold axis with the lowest surface energy will be the orientation of epitaxial film on muscovite. In most oxide epitaxial systems on muscovite, the lattice constants and properties were shown to be 


\begin{tabular}{|c|c|c|c|c|c|c|c|}
\hline & Rocksalt & Wurtzite & Perovskite & Inverse spinel & Fluorite & \multicolumn{2}{|c|}{ Others } \\
\hline Material & $\mathrm{NiO}$ & $\mathrm{ZnO}$ & $\mathrm{SrTiO}_{3}$ & $\mathrm{CoFe}_{2} \mathrm{O}_{4}$ & YSZ & $\mathrm{MoO}_{2}$ & $\mathrm{VO}_{2}$ \\
\hline $\begin{array}{l}\text { Epitaxial } \\
\text { relationship }\end{array}$ & $\begin{array}{l}\mathrm{NiO}[111]|| \text { |Mica[001] } \\
\mathrm{NiO}[11-2]|| \text { Mica[100] }\end{array}$ & $\begin{array}{l}\text { ZnO [001] ||Mica[001] } \\
\text { ZnO [100]||Mica[100] }\end{array}$ & $\begin{array}{l}\text { STO[111]|| Mica[001] } \\
\text { STO[11-2]|| Mica[100] }\end{array}$ & $\begin{array}{l}\text { CFO[111] | | Mica[001] } \\
\text { CFO[11-2]||Mica[100] }\end{array}$ & $\begin{array}{l}\text { YSZ [111] | | Mica[001] } \\
\text { YSZ [11-2]|| Mica[100] }\end{array}$ & $\begin{array}{l}\mathrm{MoO}_{2}[010]|| \text { Mica[001] } \\
\mathrm{MoO}_{2}[001]|| \text { Mica }[100]\end{array}$ & $\begin{array}{l}\mathrm{VO}_{2}[010]|| \mathrm{Mica}[001] \\
\mathrm{VO}_{2}[001]|| \mathrm{Mica}[100]\end{array}$ \\
\hline Application & RRAM & $\begin{array}{c}\text { Transparent } \\
\text { conducting oxide }\end{array}$ & High-K material & Magnetostriction & SOFC, Gas sensor & Electrode & Smart window \\
\hline
\end{tabular}

\begin{tabular}{ll}
\hline Material & Muscovite $-\mathrm{KAl}_{2} \mathrm{AlSi}_{3} \mathrm{O}_{10}(\mathrm{OH})_{2}$ \\
\hline Lattice parameters & $\mathrm{a}=0.525 \mathrm{~nm}, \alpha=90^{\circ}$ \\
& $\mathrm{b}=0.902 \mathrm{~nm}, \beta=100^{\circ}$ \\
& $\mathrm{c}=1.014 \mathrm{~nm}, \gamma=90^{\circ}$ \\
Advantages & - Transparent \\
& $\cdot$ Flexible \\
& - High thermal \& chemical stability \\
\hline
\end{tabular}

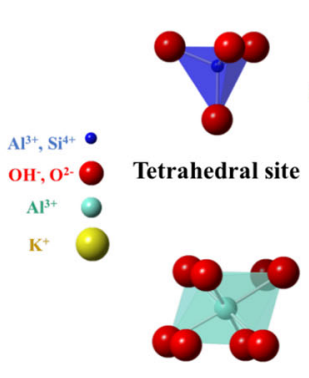

Octahedral site

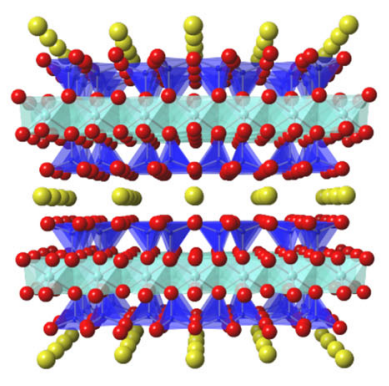

Muscovite

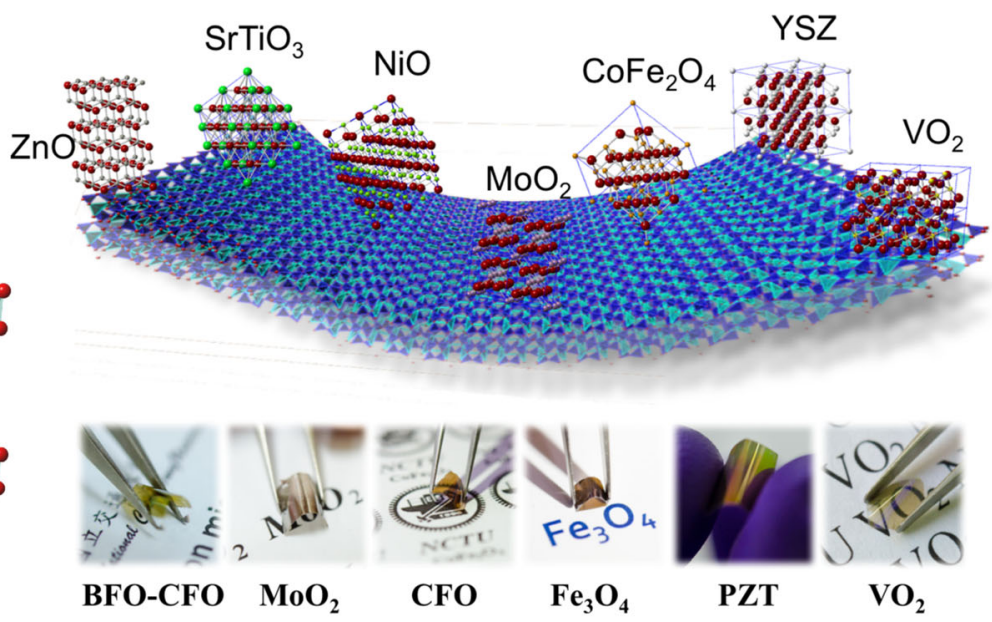

Fig. 2 A summary on the current status of vdW oxide heteroepitaxy ${ }^{31,34,36-40}$

close to bulk materials or single crystals. A good system to illustrate the characteristics of strain-free and removal of substrate clamping is the $\mathrm{CoFe}_{2} \mathrm{O}_{4} /$ muscovite system. ${ }^{34}$ The lattice parameters were found to be very close to bulk, indicating a mitigation of the strain. Since $\mathrm{CoFe}_{2} \mathrm{O}_{4}$ is a magnetostrictive material, it was found that the magnetostrictive coefficient is very close to the single crystal value, suggesting a lack of substrate clamping.

vdW heteroepitaxy on muscovite is an excellent platform to develop oxide heteroepitaxy without strain and substrate clamping. Therefore, it is very crucial to examine the characteristics of muscovite and identify key features of this type of oxide heteroepitaxy. ${ }^{27}$ Mica possesses very high yield strain and the thickness of a single cleaved mica sheet can be controlled down to few microns with superior flexibility. In fact, a bending radius down to $0.03 \mathrm{~cm}$ is reported for $100 \mathrm{~nm}$ thick mica sheet. ${ }^{35}$ Due to the weak vdW interaction, the overlayer can be peeled off easily from mica creating an unconstrained or free-standing thin film. In addition, a thin mica $(\sim 100 \mu \mathrm{m})$ sheet exhibits extraordinary transmittance in the ultraviolet-visible-infrared range of the electromagnetic spectrum. Its high melting point $(1150-1300 \mathrm{~K})$ makes it compatible with modern thin film processes. They are tolerant against chemicals for use in biomedical and wearable applications. All these features qualify mica as a destination substrate for flexible and transparent electronics.

In order to promote vdW oxide heteroepitaxy on muscovite for flexible and transparent electronics, functional materials on muscovite should be developed. Since muscovite is insulating and transparent, to build up transparent conducting oxides, serving the fundamental components in optoelectronic applications, seems to be the first step. Currently, high-quality oxide heteroepitaxy on muscovite with Indium Tin oxide and Al-doped zinc oxides are all developed, showing good flexibility, transparency, and cyclability. ${ }^{36}$ Recently, flexible $\mathrm{VO}_{2}$ /muscovite heteroepitaxy has also been demonstrated, ${ }^{37}$ owing to its metal-insulator transition in the vicinity of room temperature, delivering a material solution using correlated electrons. Furthermore, a recent study has shown the growth of epitaxial $\mathrm{Fe}_{3} \mathrm{O}_{4}$ on muscovite to advance the field of spintronics, because $\mathrm{Fe}_{3} \mathrm{O}_{4}$ is the most attractive material for such applications due to its high Curie temperature $(858 \mathrm{~K})$ and predicted nearly $100 \%$ spin polarization. ${ }^{38}$ The verification of the cyclability and endurability of $\mathrm{Fe}_{3} \mathrm{O}_{4} /$ muscovite heterostructure opens a new pathway towards flexible spintronics. Lately, there is a demonstration of the heteroepitaxy with $\mathrm{Pb}(\mathrm{Zr}, \mathrm{Ti}) \mathrm{O}_{3}$, a classic ferroelectric material, on muscovite. ${ }^{39} \mathrm{An}$ extensive exploration of device performance validated a robust ferroelectric memory device with good flexibility and cyclability. Furthermore, the heteroepitaxy on muscovite provides a solution to show excellent performance of strictive materials, such as piezoelectric $\mathrm{Pb}(\mathrm{Zr}, \mathrm{Ti}) \mathrm{O}_{3}$ and magnetostrictive $\mathrm{CoFe}_{2} \mathrm{O}_{4}$. It is very straightforward to combine them together to form a magnetoelectric composite system. A recent study based on flexible $\mathrm{BiFeO}_{3}-\mathrm{CoFe}_{2} \mathrm{O}_{4}$ heteroepitaxy on muscovite represents an important progress along this research direction since such a heterostructure can be used to optimize the performance due to a minimization of the substrate clamping effect. ${ }^{40}$ It also suggests that the research field of oxide heteroepitaxial nanocomposite on rigid substrates can be transferred to muscovite substrate, providing more materials for flexible applications. ${ }^{41,42}$ Based on these studies, more functional oxides will be implemented on muscovite epitaxially in order to expand the material database on muscovite for flexible and transparent electronics.

To sum up, vdW oxide heteroepitaxy is a new and fast-growing research direction not only for fundamental research but also for practical applications. In terms of fundamental study, there are several new research directions. (1). Since there are no dangling bonds on the surface of muscovite, one could expect the formation of new structure analog to the case of graphene and diamond. Thus, the control of competing growth mechanism is the key to this success. The test of new structures based on conventional 3D functional oxides will be implemented in the ultrathin (few unit cells) regime. (2). vdW oxide heteroepitaxy with various dimensionalities of materials including nanowires and nanodots on muscovite should be explored for potential applications. ${ }^{43}$ (3). Currently, the orientation of films in most cases follows the lowest energy of surfaces. Thus, there is no misorientation control of vdW oxide heteroepitaxy, implying an 

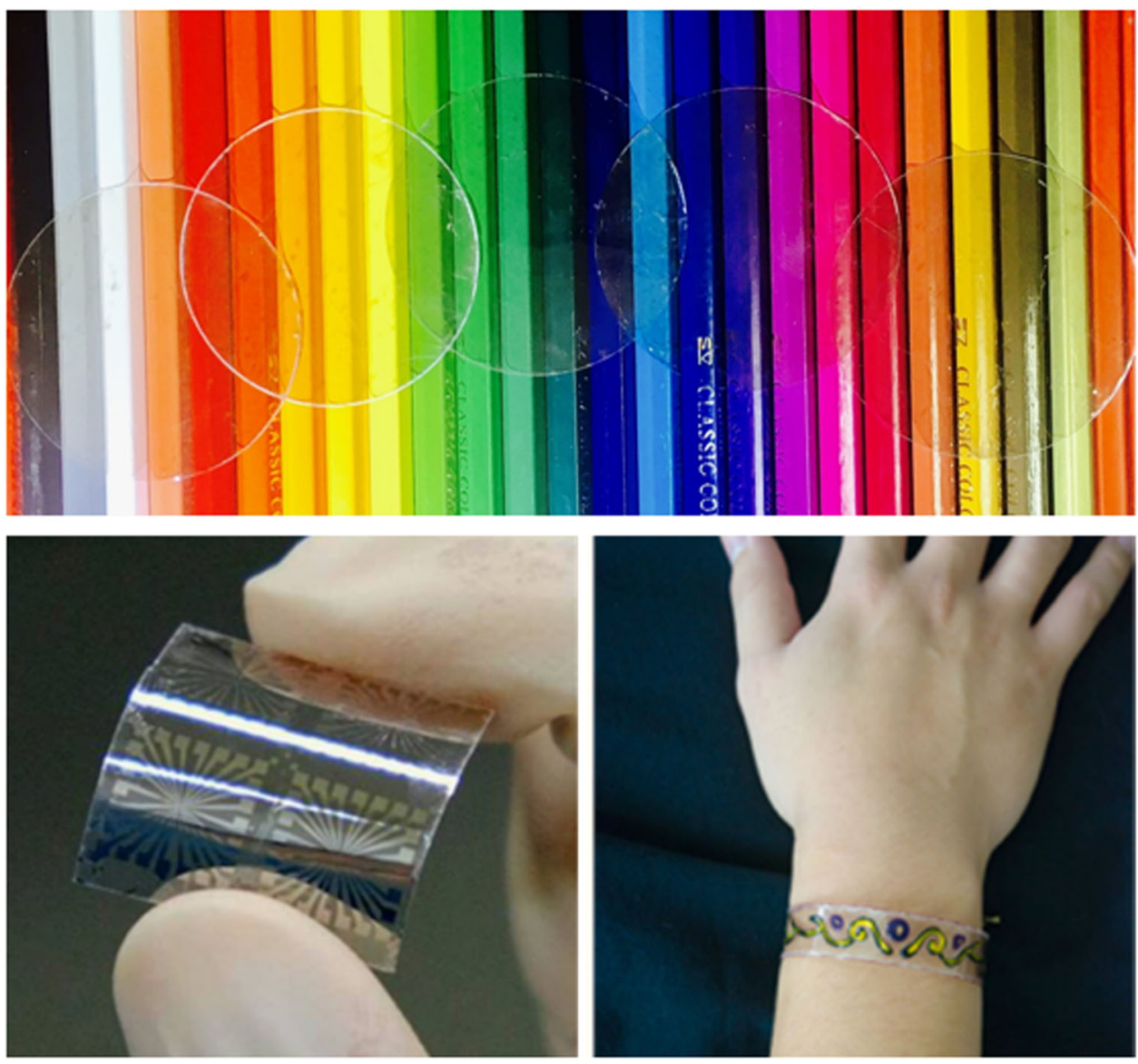

Fig. 32 " muscovite wafer, flexible electronics on muscovite, and a demonstration of wearable muscovite

important research direction since the anisotropic property is a key feature in most epitaxial thin film system. (4). In the field of strongly correlated electron systems, the interactions among degrees of freedom are mediated by the strong chemical bonds such as covalent and ionic bonds. There is no systematic study yet to understand various interactions across van der Waals gaps. However, this is very important especially for the applications of 2D materials, since the properties of 2D layered materials could be very sensitive to the environment. This may provide a new approach to tune the properties of $2 \mathrm{D}$ materials. (5). The combination of 3D functional oxides with other 2D layered materials based on the results on muscovite will also be a new research direction. In terms of practical applications, due to the flexible and transparent nature of muscovite, there are continuous research works demonstrating the possible solution for flexible electronics. $^{44,45}(6)$. New heteroepitaxy on muscovite could be developed based on other material families such as Si-, III-V, II-V, and metal-alloy-based materials, serving a new platform to integrate devices with various material combinations. (7). In the push for this research direction, a standard approach to produce muscovite wafer is needed. As shown in the top panel of Fig. 3, currently 2 " -6 " muscovite wafers can be generated in a laboratory. However, the quality of natural muscovite is not stable due to a variation of composition and the size of artificial ones is still small (less than $10 \mathrm{~cm}$ by $10 \mathrm{~cm}$ ). Thus, the production of mica substrates with large size is a challenge of manufacturing. In order to build up the fabrication chain, the design rules of fabrications should be developed to generate more architectures for device manufacturing as shown in the left-bottom panel of Fig. 3. Currently, transparent and flexible devices are dominated by polymer substrates. Identification of new applications such as wearable devices as shown in the right-bottom panel of Fig. 3 by taking the advantages of muscovite systems is crucial to overcome the barrier of entering commercial market. There are numerous opportunities and exciting challenges in the field of vdW oxide heteroepitaxy, waiting for more researchers to work together to use this new platform for future soft technology with superior mechanical, chemical, and thermal stabilities.

\section{ACKNOWLEDGEMENTS}

The author gratefully acknowledges the financial support by the Ministry of Science and Technology, R.O.C. (MOST 106-2628-E-009-001-MY2, MOST 106-2119-M-009-011MY3, and MOST 106-2218-E-009-021) and the assistances from Dr. Yugandhar Bitla, Mr. Chun-Hao Ma, Mr. Min Yen, Ms. Pei-Chun Wang, and Ms. I-Hsuan Lin.

\section{AUTHOR CONTRIBUTIONS}

Y.H.C. conceived and wrote the paper.

\section{ADDITIONAL INFORMATION}

Competing interests: The author declares that he has no competing financial interests.

Publisher's note: Springer Nature remains neutral with regard to jurisdictional claims in published maps and institutional affiliations.

\section{REFERENCES}

1. Takagi, H. \& Hwang, H. Y. An emergent change of phase for electronics. Science 327, 1601-1602 (2010).

2. Mannhart, J. \& Schlom, D. G. Oxide interfaces-an opportunity for electronics. Science 327, 1607-1611 (2010). 
3. Ueda, K., Tabata, H. \& Kawai, T. Ferromagnetism in $\mathrm{LaFeO}_{3}-\mathrm{LaCrO}_{3}$ superlattices. Science 280, 1064-1066 (1998).

4. Zheng, $\mathrm{H}$. et al. Multiferroic $\mathrm{BaTiO}_{3}-\mathrm{CoFe}_{2} \mathrm{O}_{4}$ nanostructures. Science 303, 661-663 (2004).

5. Macmanus-driscoll, J. L. et al. Strain control and spontaneous phase ordering in vertical nanocomposite heteroepitaxial thin films. Nature Mater. 7, 314-320 (2008).

6. Schlom, D. G., Chen, L. Q., Pan, X. Q. \& Zurbuchen, M. A. A thin film approach to engineering functionality into oxides. J. Am. Ceram. Soc. 91, 2429-2454 (2008).

7. Zeches, R. J. et al. A strain-driven morphotropic phase boundary in $\mathrm{BiFeO}_{3}$. Science 326, 977-980 (2009).

8. Wong, W. S., Sands, T. \& Cheung, N. W. Damage-free separation of GaN thin films from sapphire substrates. Appl. Phys. Lett. 72, 599 (1998).

9. Tsakalakos, L. \& Sands, T. Epitaxial ferroelectric $(\mathrm{Pb}, \mathrm{La})(\mathrm{Zr}, \mathrm{Ti}) \mathrm{O}_{3}$ thin films on stainless steel by excimer laser liftoff. Appl. Phys. Lett. 76, 227 (2000).

10. Nagarajan, V. et al. Dynamics of ferroelastic domains in ferroelectric thin films. Nature Mater. 2, 43-47 (2003).

11. Zhang, J. X. et al. A nanoscale shape memory oxide. Nature Commun. 4, 2768 (2013).

12. Tian, G. et al. Magnetoelectric coupling in well-ordered epitaxial $\mathrm{BiFeO}_{3} / \mathrm{CoFe}_{2} \mathrm{O}_{4} /$ $\mathrm{SrRuO}_{3}$ heterostructured nanodot array. ACS Nano 10, 1025-1032 (2016).

13. Bakaul, S. R. et al. Single crystal functional oxides on silicon. Nature Commun. 7, 10547 (2017).

14. Lu, D. et al. Synthesis of freestanding single-crystal perovskite films and heterostructures by etching of sacrificial water-soluble layers. Nature Mater. 15, 1255-1261 (2017).

15. Zhang, Y. et al. Flexible quasi-two-dimensional $\mathrm{CoFe}_{2} \mathrm{O}_{4}$ epitaxial thin films for continuous strain tuning of magnetic properties. ACS Nano 11, 8002-8009 (2017).

16. Koma, A. \& Yoshimura, K. Ultrasharp interfaces grown with van der Waals epitaxy. Surf. Sci. 174, 556-560 (1986)

17. Koma, A., Ueno, K. \& Saiki, K. Heteroepitaxial growth by van der Waals interaction in one-, two- and three-dimensional materials. J. Cryst. Growth 111, 1029-1032 (1991).

18. Koma, A. Van der Waals epitaxy-a new epitaxial growth method for a highly lattice-mismatched system. Thin Solid Films 216, 72-76 (1992).

19. Koma, A. Van der Waals epitaxy for highly lattice-mismatched systems. J. Cryst. Growth 201-202, 236-241 (1999).

20. Geim, A. K. \& Grigorieva, I. V. Van der Waals heterostructures. Nature 499, 419-425 (2013)

21. Kim, J. et al. Principle of direct van der Waals epitaxy of single-crystalline films on epitaxial graphene. Nature Commun. 5, 4836 (2014).

22. Novoselov, K. S., Mishchenko, A., Carvalho, A. \& Castro Neto, A. H. 2D materials and van der Waals heterostructures. Science 353, aac9439 (2016).

23. Jariwala, D., Marks, T. J. \& Hersam, M. C. Mixed-dimensional van der Waals heterostructures. Nature Mater. 16, 170-181 (2017).

24. Utama, M. I. B., Mata, de la, M., Magen, C., Xiong, Q. \& Arbiol, J. Twinning-, polytypism-, and polarity-induced morphological modulation in nonplanar nanostructures with van der Waals epitaxy. Adv. Funct. Mater. 23, 1636-1646 (2013).

25. Li, M. Y. et al. Epitaxial growth of a monolayer $\mathrm{WSe}_{2}-\mathrm{MoS}_{2}$ lateral p-n junction with an atomically sharp interface. Science 349, 524-528 (2015).

26. Chiang, Y. M., Birnie III, D. \& Kingery, W. D. Physical Ceramics: Principles for Ceramic Science and Engineering. (John Wiley \& Sons, Inc, Canada, 1997).

27. Bitla, Y. \& Chu, Y. H. MICAtronics: a new platform for flexible X-tronics. FlatChem 3, 26-42 (2017).
28. Steinberg, S. et al. Van der Waals epitaxial growth of alpha-alumina nanocrystals on mica. Science 260, 656-659 (1993).

29. Hines, H. L. Epitaxial growth of gold on mica in an ultra-high vacuum. Journal de Physique 25, 134-137 (1964).

30. Wang, K. et al. High-quality $\mathrm{Bi}_{2} \mathrm{Te}_{3}$ thin films grown on mica substrates for potential optoelectronic applications. Appl. Phys. Lett. 103, 031605 (2013).

31. $\mathrm{Ma}, \mathrm{C}$. H. et al. Van der Waals epitaxy of functional $\mathrm{MoO}_{2}$ film on mica for flexible electronics. Appl. Phys. Lett. 108, 253104 (2016).

32. Ke, S. et al. Transparent indium tin oxide electrodes on muscovite mica for hightemperature-processed flexible optoelectronic devices. ACS Appl. Mater. Interfaces 8, 28406-28411 (2016).

33. Gao, W. et al. Flexible $\mathrm{PbZr}_{0.52} \mathrm{Ti}_{0.48} \mathrm{O}_{3}$ capacitors with giant piezoelectric response and dielectric tunability. Adv. Electron. Mater. 3, 1600542 (2017).

34. Liu, H. J. et al. Flexible heteroepitaxy of $\mathrm{CoFe}_{2} \mathrm{O}_{4} /$ Muscovite bimorph with large magnetostriction. ACS Appl. Mater. Interfaces 9, 7297-7304 (2017).

35. He, Y. et al. Mica, a potential two-dimensional-crystal gate insulator for organic field-effect transistors. Adv. Mater. 23, 5502-5507 (2011).

36. Bitla, Y. et al. Oxide heteroepitaxy for flexible optoelectronics. ACS Appl. Mater. Interfaces 8, 32401-32407 (2016).

37. Li, C. I. et al. Van der Waals epitaxy of flexible and transparent $\mathrm{VO}_{2}$ film on muscovite. Chem. Mater. 28, 3914-3919 (2016).

38. Wu, P. C. et al. Heteroepitaxy of $\mathrm{Fe}_{3} \mathrm{O}_{4}$ /muscovite: a new perspective for flexible spintronics. ACS Appl. Mater. Interfaces 8, 33794-33801 (2016).

39. Amrillah, T. et al. Flexible multiferroic bulk heterojunction with giant magnetoelectric coupling via van der Waals epitaxy. ACS Nano 11, 6122-6130 (2017).

40. Jiang, J. et al. Flexible ferroelectric element based on van der Waals heteroepitaxy. Sci. Adv. 3, e1700121 (2017).

41. Liu, H. J., Liang, W. I., Chu, Y. H., Zheng, H. M. \& Ramesh, R. Self-assembled vertical heteroepitaxial nanostructures: from growth to functionalities. MRS Commun. 4 167-181 (2014).

42. Yang, J. C., Liu, H. J. \& Chu, Y. H. Mesocrystal-embedded functional oxide systems. MRS Commun. 6, 31-44 (2016).

43. Utama, M. I. B. et al. Incommensurate van der Waals epitaxy of nanowire arrays: a case study with ZnO on muscovite mica substrates. Nano Lett. 12, 2146-2152 (2012).

44. Li, M., Wang, Y., Wang, Y. \& Wei, X. AZO/Ag/AZO transparent flexible electrodes on mica substrates for high temperature application. Ceram. Int. 43, 15442-15446 (2017).

45. Yang, Y. et al. Flexible, semitransparent, and inorganic resistive memory based on $\mathrm{BaTi}_{0.95} \mathrm{Co}_{0.05} \mathrm{O}_{3}$ film. Adv. Mater. 29, 1700425 (2017).

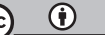

Open Access This article is licensed under a Creative Commons Attribution 4.0 International License, which permits use, sharing, adaptation, distribution and reproduction in any medium or format, as long as you give appropriate credit to the original author(s) and the source, provide a link to the Creative Commons license, and indicate if changes were made. The images or other third party material in this article are included in the article's Creative Commons license, unless indicated otherwise in a credit line to the material. If material is not included in the article's Creative Commons license and your intended use is not permitted by statutory regulation or exceeds the permitted use, you will need to obtain permission directly from the copyright holder. To view a copy of this license, visit http://creativecommons. org/licenses/by/4.0/.

(c) The Author(s) 2017 